
\title{
28 Research Square \\ Vitamin D deficiency in critically ill patients diagnosed with COVID -19. Are we doing enough? A retrospective analysis of 226 patients.
}

Tomás Cuñat ( $\nabla$ cunat@clinic.cat)

Hospital Clínic de Barcelona

Antonio Ojeda

Hospital Clínic de Barcelona

Andrea Calvo

Hospital Clínic de Barcelona

\section{Short Report}

Keywords: Vitamin D, immunomodulation, intensive care, critical care, COVID-19, SARS-COV2.

Posted Date: May 22nd, 2020

DOI: https://doi.org/10.21203/rs.3.rs-30390/v1

License: (c) (i) This work is licensed under a Creative Commons Attribution 4.0 International License.

Read Full License 


\section{Abstract}

Vitamin D deficiency is common in critically ill patients, and its role in COVID-19 patients could be important. Its deficiency has been associated with respiratory distress syndrome, pulmonary fibrosis (through activation of the renin-angiotensin system), increased levels of IL-2, and cardiovascular adverse events. Various scientific societies recommend the screening of vitamin $D$ in individuals at risk for deficiency. Despite that, the demographics of Vitamin D levels amongst critically ill patients with a confirmed diagnosis of COVID-19 are currently unknown. We propose a study to determine the prevalence of vitamin D deficiency in a consecutive population of COVID-19 patients admitted to intensive care units and to evaluate its relationship with clinical outcomes. We study 226 COVID-19 patients between March 16 and April 26, 2020. The prevalence of vitamin D deficiency could not be determined because the value of 25-hydroxyvitamin D was obtained in a few patients (17 patients, 7,5\%). However, all patients with serum determinations of 25-hydroxyvitamin D presented a level lower than $20 \mathrm{ng} / \mathrm{ml}$ and thirteen patients $(76,5 \%)$ levels $<12,5 \mathrm{ng} / \mathrm{ml}$. We conclude that undiagnosed vitamin D deficiency is common in critically ill COVID-19 patients, and physicians should be conscious of the relevance of its monitoring and supplementation.

\section{Manuscript Text}

The novel coronavirus infection (COVID-19), first identified in December 2019 in Wuhan, China, has contributed to significant mortality in several countries with the number of infected cases increasing exponentially worldwide. Therefore, the search for possible strategies that allow reducing the number of infected or influencing the main immunological mechanisms of the virus, is one of the main therapeutic approaches.

Vitamin D deficiency in critically ill patients has been reported for years in different diseases and patient populations, and it's been associated with worse clinical outcomes. Critical COVID-19 patients, have common risk factors (comorbidities, inadequate intake, absence of sun exposure and immobilization), frequently received antiretrovirals therapy, that increase the catabolism of this vitamin.[1]

Interest in the role of vitamin D in COVID-19 has increased in the last months because vitamin D is essential in several mechanisms to reduce infections (physical barrier, innate and adaptive immunity) and to modulate excessive inflammation.[2] Moreover, its deficiency has been associated with respiratory distress syndrome, pulmonary fibrosis through activation of the renin-angiotensin system (RAS), and increased levels of IL-2. In critically ill patients it is also important to mention the role of vitamin D in the cardiovascular system and its association with myopathy, and both situations increase the likelihood of complications during intensive care unit (ICU) stay.[3]

Various scientific societies recommend the screening of vitamin D in individual at risk for deficiency and although there is no global consensus, the European Society for Clinical Nutrition and Metabolism 
(ESPEN) recommends to supplement vitamin D In critically ill patients if plasma measured levels are low (25-hydroxy-vitamin $\mathrm{D}<12.5 \mathrm{ng} / \mathrm{ml}$, or $50 \mathrm{nmol} / \mathrm{l}$ ). [4]

Despite that, to our knowledge, the demographics of Vitamin D levels amongst critically ill patients with a confirmed diagnosis of COVID-19 are currently unknown.

The aim of our study was to evaluate the prevalence of vitamin D deficiency in a consecutive population of COVID-19 patients admitted to intensive care units of a single center and to evaluate its relationship with clinical outcomes.

We performed a retrospective transversal study, adults patients diagnosed with COVID-19 infection according to World Health Organization criteria, consecutively admitted to the ICU from Hospital Clínic of Barcelona, Spain, between March 16 and April 26, 2020.

The study was approved by the Ethics Committee of HCB with approval number HCB/2020/0580 in accordance with the Helsinki Declaration of 1975.

Through electronic medical record, we collected the level of serum 25-Hydrovitamin D, parathormone (PTH), total serum proteins, calcium, and phosphate during ICU stay with central core laboratory head assessment.

The measurement method used for the assessment of 25 -Hydrovitamin D was by an automated chemiluminescence immunoassay with a Liaison analyzer ${ }^{\mathrm{TM}}$ (Diasorin, Saluggia, Italy).

Vitamin D deficiency was defined as a 25-hydroxyvitamin D level $<20 \mathrm{ng} / \mathrm{ml}$. According to ESPEN guidelines a 25 -hydroxyvitamin $D<12.5 \mathrm{ng} / \mathrm{ml}$ was cut-off to considerate that vitamin $D$ has to be supplemented.[4]

Hypocalcemia and hypercalcemia were defined as serum calcium levels less than $8.5 \mathrm{mg} / \mathrm{dL}$ and more than $10.6 \mathrm{mg} / \mathrm{dL}$ respectively. Corrected calcium was calculated by measured total calcium / $(0,6+$ (total proteins/18,5)).

Hypophosphatemia and hypophosphatemia were defined as serum phosphate levels less than $3.5 \mathrm{mg} / \mathrm{dL}$ and more than $4.5 \mathrm{mg} / \mathrm{dL}$, respectively. Elevated PTH level was defined as a serum PTH level more than $65 \mathrm{pg} / \mathrm{mL} .[5]$

All critically ill patients were characterized by sex, race, chronic renal failure, and Charlson index. APACHE II and SOFA were registered at admission. Requirements of mechanical ventilation and days of therapy, use of corticosteroids, antiretroviral therapy, hospital-acquired infections, ICU length of stay, mortality, and vitamin $\mathrm{D}$ and calcium metabolism analysis were collected.

A descriptive analysis was performed using Statistical Package for Social Sciences version 22.0 (SPSS, Chicago, IL). Data are presented in number and proportions (\%) for categorical variables and mean and standard deviation (SD) for continuous variables. 
We evaluated 226 adult patients diagnosed with COVID-19 infection. Basal demographical data and ICU outcomes of these patients are shown in Table 1.

Fifty-eight percent of patients were men, and median age was 64 years. Median SOFA was $10(3,28)$, mainly at the expense of respiratory failure. Seventy-six percent of patients presented a nosocomial infection during the stay at ICU. Seventeen patients $(7,5 \%)$ had at least one Vitamin D serum determination.

The prevalence of vitamin $D$ deficiency could not be determined because the value of 25-hydroxyvitamin D was obtained in very few patients. However, all patients with 25 -hydroxyvitamin D serum determination presented a value lower than $20 \mathrm{ng} / \mathrm{ml}$.

Thirteen patients $(76.5 \%)$ had 25-hydroxyvitamin D levels $<12.5 \%$, with 3 patients with values lower than $5 \mathrm{ng} / \mathrm{ml}$. Hypocalcemia and hypophosphatemia were observed in $6(35.2 \%)$ and 11 (64.7\%) patients, respectively. All patients had elevated PTH levels (Table 2).

Despite significant and recent scientific evidence of the vitamin D role on critical illness, only $7.5 \%$ of patients admitted to the ICU had at least one measurement of vitamin D levels during the stay at ICU. Vitamin $D$ measurements have not been adequately included as a part of the routine tests to be evaluated in patients admitted at ICU an even less so in patients diagnosed with COVID -19. These findings could suggest that, if there were a high vitamin D deficiency in patients admitted to the ICU, only a small percentage could be diagnosed and supplemented. We propose a different hypothesis to explain the low request of vitamin $D$, lack of knowledge, skepticism of physicians, and the absence of a global consensus regarding the importance of treatment vitamin D deficiency in critical care illness.

Given the retrospective nature of the study, it is not possible to determine whether the high observed proportion of patients with hypocalcemia $(35.2 \%)$ and hypophosphatemia $(64.7 \%)$ is a consequence of hypovitaminosis $D$ and which is its clinical significance. However, it is known that they are related to arrhythmias and impaired myocardial contractility, metabolic encephalopathy, proximal myopathy, and respiratory failure. These clinical manifestations are also frequent in COVID-19 disease in critically ill patients.[1]

A high incidence of nosocomial infections (76.5\%), was observed (Table 1), vitamin D deficiency could have an important role in their development reducing concentrations of pro-inflammatory cytokines, as well as increasing concentrations of anti-inflammatory cytokines.[2]

Our study has several limitations, due to the retrospective nature of the study, only one 25-hydroxyvitamin D serum determination was obtained, and it corresponded to different days in the course of the disease for all patients. Consequently, it difficulted any correlation with the evolution of the disease. Additionally, the previous vitamin D deficit was unknown.

So far, studies showing the relationship between vitamin $D$ deficiency and the progression of the disease due to COVID-19 are lacking. 
Likewise, the interest in optimizing the levels of vitamin $D$ as well as other vitamins and minerals, as part of prevention both in health workers and in the population continues to grow.

To conclude, undiagnosed vitamin D deficiency is common in critically ill COVID-19 patients, and physicians should be conscious of the relevance of monitoring and supplementing when it is present.

\section{Declarations}

Funding: This research did not receive any specific grant from funding agencies in the public, commercial, or not-for-profit sectors.

\section{Acknowledgements}

The authors thank to Dr. Xavier Filella from the Department of Biochemistry and Molecular Genetics, Hospital clinic of Barcelona for his help in collecting the data, for this study.

Competing interests: The authors declare no competing interests.

The ethics committee waived the need for informed consent as patients are no longer visited periodically and are not viable.

\section{References}

[1]. Putzu A, Belletti A, Cassina T, et al. Vitamin D and outcomes in adult critically ill patients. A systematic review and meta-analysis of randomized trials. J Crit Care. 2017;38:109-114. https://doi:10.1016/j.jcrc.2016.10.029

[2]. Grant WB, Lahore H, McDonnell SL, et al. Evidence that Vitamin D Supplementation Could Reduce Risk of Influenza and COVID-19 Infections and Deaths. Nutrients. 2020;12(4). https://doi:10.3390/nu12040988

[3]. Ma D, Peng L. Vitamin D and pulmonary fibrosis: a review of molecular mechanisms. Int $J$ Clin Exp Pathol. 2019;12(9):3171-3178.

[4]. Singer $P$, Blaser $A R$, Berger MM, et al. ESPEN guideline on clinical nutrition in the intensive care unit. Clin Nutr. 2019;38(1):48-79. https://doi:10.1016/j.clnu.2018.08.037

[5]. Ardehali SH, Dehghan S, Baghestani AR, Velayati A, Vahdat Shariatpanahi Z. Association of admission serum levels of Vitamin $D$, calcium, Phosphate, magnesium and parathormone with clinical outcomes in neurosurgical ICU patients. Sci Rep. 2018;8(1):1-8. https://doi:10.1038/s41598-018-21177-4

\section{Tables}

Table 1 Baseline patient characteristics and ICU outcomes of patients with 250h measurement. 


\begin{tabular}{|c|c|}
\hline & $\mathrm{n}=17$ \\
\hline Age (years) & $64.94(10.69)$ \\
\hline \multirow[t]{2}{*}{ Sex } & Female: $7(41.2 \%)$ \\
\hline & Male: $10(58.8 \%)$ \\
\hline Smoke History & $4(23.5 \%)$ \\
\hline BMI (kg/m2) & $29.11(3.93)$ \\
\hline Charlson Index score & $2.8(1.81)$ \\
\hline Chronic Kidney Disease & $2(11.8 \%)$ \\
\hline APACHE II & $16.59(7.81)$ \\
\hline SOFA score & $10.53(3.28)$ \\
\hline Mechanical ventilation & $15(88.2 \%)$ \\
\hline Mechanical ventilation (days) & $18(12.4)$ \\
\hline ICU acquired weakness & $16(94.1 \%)$ \\
\hline Lenght of ICU stay & $27.88(10.39)$ \\
\hline Altered liver function & $8(47.1 \%)$ \\
\hline xAnti-Retroviral Drugs & $17(100 \%)$ \\
\hline High-dose Steroid & $16(94.1 \%)$ \\
\hline Nosocomial infection & $13(76.5 \%)$ \\
\hline Pneumonia & $4(23.5 \%)$ \\
\hline Urinary Tract Infection & $2(11.8 \%)$ \\
\hline Catheter-related Bloodstream Infection & $7(41.2 \%)$ \\
\hline Septic Shock & $6(35.3 \%)$ \\
\hline Mortality & $1(5.9 \%)$ \\
\hline
\end{tabular}

Data are in number and proportions (\%) for categorical variables and in mean and standard deviation (SD) for continuous variables.

Abbreviations: BMI: Body Mass Index; Charlson Index score: Charlson Combined Comorbidity Index score; APACHE II: Acute Physiology and Chronic Health Evaluation II; SOFA: Sequential Organ Failure Assessment; ICU:Intensive Care Unit.

Table 2 Vitamin D and mineral metabolism related variables 


\begin{tabular}{|c|c|}
\hline & $\mathrm{n}=17$ \\
\hline Total Serum Calcium(mg/dL) & $7.9(0.76)$ \\
\hline Corrected total serum Calcium $(\mathrm{mg} / \mathrm{dL})$ & $8.8(0.75)$ \\
\hline Serum Posphate $(\mathrm{mg} / \mathrm{dL})$ & $3.723(1.04)$ \\
\hline Total serum Proteins $(\mathrm{g} / \mathrm{L})$ & $54.76(4.94)$ \\
\hline Serum creatinine $(\mathrm{mg} / \mathrm{dL})$ & $0.89(0.59)$ \\
\hline 25-Hydrovitamin D $(\mathrm{ng} / \mathrm{mL})$ & $9.98(4)$ \\
\hline 25-Hydrovitamin D $<20 \mathrm{ng} / \mathrm{mL}$ & $17(100 \%)$ \\
\hline 25-Hydrovitamin $\mathrm{D}<12,5 \mathrm{ng} / \mathrm{ml}$ & $13(76,5 \%)$ \\
\hline Parathormone $(\mathrm{pg} / \mathrm{mL})$ & $116(97.63)$ \\
\hline
\end{tabular}

Data are in number and proportions (\%) for categorical variables and in mean and standard deviation (SD) for continuous variables. 